\title{
OPTIMAL MULTIOBJECTIVE DESIGN OF DIGITAL FILTERS USING TAGUCHI OPTIMIZATION TECHNIQUE
}

\author{
Abderrahmane Ouadi — Hamid Bentarzi — Abdelmadjid Recioui
}

\begin{abstract}
The multiobjective design of digital filters using the powerful Taguchi optimization technique is considered in this paper. This relatively new optimization tool has been recently introduced to the field of engineering and is based on orthogonal arrays. It is characterized by its robustness, immunity to local optima trapping, relative fast convergence and ease of implementation. The objectives of filter design include matching some desired frequency response while having minimum linear phase; hence, reducing the time response. The results demonstrate that the proposed problem solving approach blended with the use of the Taguchi optimization technique produced filters that fulfill the desired characteristics and are of practical use.

K e y w or d s: multiobjective filter design, Taguchi optimization technique, magnitude response, minimum linear phase, group delay
\end{abstract}

\section{INTRODUCTION}

Digital filters exist in two types: Finite impulse response (FIR) and Infinite impulse response (IIR) or recursive. FIR filters suffer from the problem of high order (hence implementation and performance issues) if strict requirements are imposed at the design stage. Furthermore, IIR filters can have smaller group delay than its equivalent FIR filters $[1,2]$. The optimal design of an infinite impulse response (IIR) filter consists in choosing a set of coefficients of the filter to have a frequency response that optimally approximates the desired response [1-22].

Different techniques exist for the design of digital filters. Windowing method; in which the ideal impulse response is multiplied by a window function, is the most popular. There are various kinds of window functions (Butterworth, Chebyshev, Kaiser etc), depending on the requirements on ripples in the passband and stopband, stopband attenuation and the transition width. These various windows limit the infinite length impulse response of ideal filter into a finite window to design an actual response. Furthermore, windowing methods do not allow sufficient control of the frequency response in the various frequency bands and other filter parameters such as transition width. The designer always has to compromise between the design specifications $[1,2]$.

Due to the presence of the denominator of the transfer function, the stability condition of the filter should be taken into account in the optimal design $[1,2-7,9-18,11-$ $18,20,22,23]$, resulting in a constrained optimization problem. Several sufficient conditions $[2-4,6,10,12,18]$ have been established for the parameterization that represents the filters denominator by a single polynomial. The triangle-based stability conditions [1] are necessary and sufficient and have been incorporated into several design procedures $[11,13,14]$ that formularize the filters denominator by cascaded second-order sections (SOSs).
In [13], variable transformation is used to convert the finite stability region into the entire coefficient space, such that the original constrained design problem becomes an unconstrained one in the transformed space. However, the transformation increases the nonlinearity of the objective function, which makes it hard to find good (global optimum) solutions in general. In [14], a perturbed stability triangle is proposed to guarantee the SOS to have its zeroes inside a circle of given radius. It is combined with the Gauss-Newton strategy, resulting in an improved design. In [15], the conditions presented for the SOS with zeroes inside a circle of given radius enclose a triangular stability domain and can be easily incorporated into any constrained optimization formulations based on the SOS parameterization. A method that divides the overall design of an IIR filter into successive designs of its secondorder sections is presented in [20], where one section is first designed, and then, another section is appended until all sections are designed.

Because of finite word length effects occurring in practical implementations of the designed filters, not only stability of the filter is of great importance but a stability margin is necessary as well. The poles of the transfer function should not lie too close to the unit circle. The sensitivity of pole locations to coefficient quantization increases with decreasing distance from the unit circle. Poles close to the unit circle may considerably enhance quantization noise and increase the maximum amplitude of small scale limit cycle. Consequently, it is desirable to have control over the maximum radius when designing IIR filters.

Linear-phase filters are usually designed as non-recursive (FIR) filters which can have constant group delay over the entire baseband. However, when highly selective filters are required, a very high filter order is needed which makes these filters uneconomical or impractical. To eliminate this problem, attempts have been made to develop

* Laboratory signals and systems, Institute of Electrical and Electronic Engineering, University Mohamed Bougara de Boumerdes, Algeria. Avenue de l'indpendance, 35000, Boumerdes. Algeria, a_recioui@umbb.dz 
methods to design recursive (IIR) filters whose delay characteristics approximate a constant value in the passband. This includes IIR filter design approach that can satisfy both magnitude and phase characteristics simultaneously [24-28]. The design of IIR filters with constant group delay in the passband is also carried out by using allpass structures through evaluation of phase response instead of approximating the group delay directly [29-32]. Some other methods used an indirect approach based on model reduction techniques where a linear-phase FIR filter that meets the required specifications is first designed and then a lower order IIR filter that meets the original amplitude specifications while maintaining a linear-phase response in the passband is obtained [33-35]. Coretlazzo et al [36] have achieved the simultaneous design in both magnitude and group delay of IIR and FIR filters based on multiple criterion optimizations. Lutovac et al [37] have developed a new design method for elliptic IIR filters that provide the implementation of half of the multiplication constants with few shifters and adders. Sullivan et al [38] have proposed the algorithm based on the peakconstrained leastsquares optimality criterion for cascaded IIR filters, which can design a filter that has an equalized group delay without the use of all pass filters, and it can simultaneously meet the frequency response magnitude specifications by using all of the filter coefficients available to optimize the filter. Lang [39] has used least square method for designing IIR filter with prescribed magnitude and phase response . This parameterization of the transfer function has been used for designing IIR filters. Gordana Javanovic [40] has proposed a method for the design of IIR notch filters with desired magnitude characteristic, which can be either maximally flat or equiripple. Xi Zhang [41] have proposed a novel method for designing maximally flat IIR filters with flat group delay responses in the passband.

Under these circumstances, evolutionary and metaheuristic optimization methods find their place. These are referred to as global optimizers while the more familiar, traditional techniques such as conjugate gradient and the quasi-Newtonian methods are classified as local optimizers. The distinction between local and global search of optimization techniques is that the local techniques produce results that are highly dependent on the starting point or initial guess, while the global methods are totally independent of the initial conditions [42]. Though they possess the characteristic of being fast in convergence, local techniques, in particular the quasi-Newtonian techniques have direct dependence on the existence of at least the first derivative. In addition, they place constraints on the solution space such as differentiability and continuity; conditions that are hard or even impossible to satisfy in some situations [42].

Previously, global optimization techniques have been implemented in the design of digital filters. One such approach using neural networks has been described in [43]. Also, use of PSO in the design of frequency sampling finite impulse response (FIR) filter has been described in [44-46]. Differential evolution has been used in the design of digital filters has been implemented in [47-50].
$[51,52]$ have used Hierarchical Genetic Algorithms to design and optimization of IIR filter structures. Use of Particle Swarm Optimization (PSO) and Genetic Algorithms (GA) in the design of digital filters is described in [53].

In this work, the application of the new optimization technique called Taguchi optimization to the design of digital filters is considered. The purpose is to design a filter that can simultaneously satisfy multiobjective criteria including frequency response and linear phase with the least possible group delay.

\section{PROBLEM FORMULATION}

Digital filters find their applications in different areas. One area is power system protection where measurement systems involve faulted signals associated with DC decaying signals, harmonic and sub-harmonic components. To eliminate these unwanted components, a digital filter design based on multi-objective optimization technique to satisfy different specifications such as high speed response for a real-time application and frequency domain requirements.

\subsection{Digital Filtering Approach}

A digital filter based solution is proposed to remove unwanted disturbances using digital filter design techniques. The filter time response must be included in the requirements. The present filtering application imposes different kind of specifications. On one hand, the time domain requirement where both a high speed and accurate system response are needed. On the other hand, the frequency domain requirements (DC, sub-synchronous and harmonic components elimination) which are the magnitude response within small bandwidth including sharp frequency edges as well as an approximately constant group delay in this band are required too. Usually the best optimum value of all the objective functions of this filter design can be obtained for some values of design variables. A compromise or a trade-off between the objective functions must be made to achieve a satisfactory filter design.

The considered recursive digital filter must satisfy three multi-objective functions. These functions are: 1) meet a specified or a desired magnitude response specification; 2) an approximately constant group delay; and 3) a minimum time response or settling time which involves a minimum phase or a group delay. The optimization approach considers the discrete-time transfer function which is formulated on the basis of some desired amplitude response and a stability margin parameter. A norm of the weighted error function is then minimized with respect to the transfer-function coefficients with a prescribed maximum pole radius referred to as stability margin . The stability margin parameter is varied to optimize the filter coefficients which minimizes mainly the magnitude response, satisfies the best approximately constant group delay and the lowest group delay that leads 


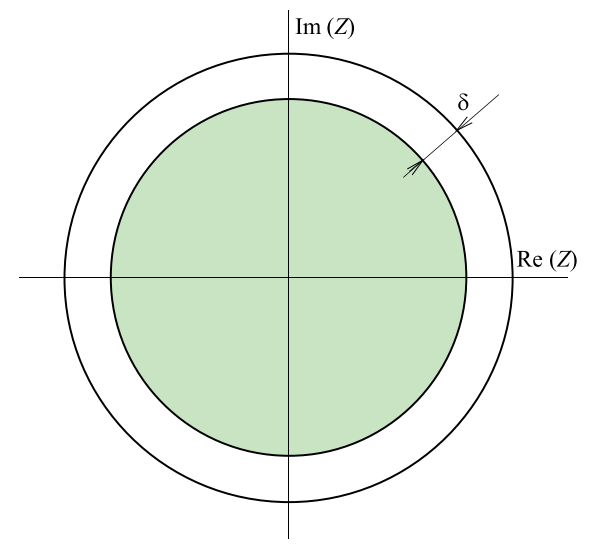

Fig. 1. Stability region and stability margin parameter $\delta$

to minimum settling time or time delay of the system dynamic response

\subsection{Filter transfer functions}

In the general case an IIR filter can be described by its discrete-time difference equation

$$
y[n]+\sum_{i=1}^{2 N-1} c_{i} y[n-1]=\sum_{j=0}^{2 M} d_{j} x[n-j]
$$

where $x(n)$ and $y(n)$ are discrete-time input and output signals. Equation (1) can be transformed into the $Z$-domain and assuming $c_{i}$ and $d_{i}$ are real coefficients a second order form transfer function can be obtained, having $2 M$ conjugate zeros and $2 N$ conjugate poles; called second order sections (SOS), as

$$
H(z)=H_{0} \frac{\prod_{j=1}^{2 M}\left(a_{0 j}+a_{1 j} z+z^{2}\right)}{\prod_{j=1}^{2 N}\left(b_{0 j}+b_{1 j} z+z^{2}\right)}
$$

where $a_{i j}$ and $b_{i j}$ are real coefficients and $H_{0}$ is a positive multiplier constant. The polar formulation is also useful and is written as

$$
H(z)=H_{0} \frac{\prod_{j=1}^{2 M}\left(z-r_{a j} e^{j \theta_{a j}}\right)\left(z-r_{a j} e^{-j \theta_{a j}}\right)}{\prod_{j=1}^{2 N}\left(z-r_{b j} e^{j \theta_{b j}}\right)\left(z-r_{b j} e^{-j \theta_{b j}}\right)}
$$

where $r_{a j}, \theta_{a j}$ and $r_{b j}, \theta_{b j}$ are the radii and angles of the zeros and poles, respectively.

\subsection{Filter stability margin}

As poles are moved toward the origin (hence decreasing the pole radius), the system stability margin parameter increases and the system settling time decreases. This action, in fact, brings two required and important properties to the designed system. First, the system time or dynamic response is enhanced as settling time is decreased. Second, the system stability becomes more robust which is a very useful property, particularly in practical implementation. Indeed, the rounding or truncation of the filter coefficients may lead to an unstable implementation if the stability margin is too small. It is therefore desirable to approximate a given response by a transfer function with a prescribed maximum pole radius named stability margin as shown in Fig. 1.

\subsection{Magnitude response objective function}

The amplitude and the phase responses of a recursive filter is given by

$$
H(z)=\left|H\left(e^{j \omega t}\right)\right|, \quad \varphi(x, \omega)=\arg \left(H\left(e^{j \omega t}\right)\right)
$$

where $\omega$ is the frequency and $x$ is a column vector with $2 M+2 N+1$ components, that is in Cartesian form

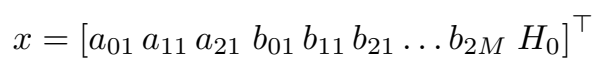

or in polar form

$$
x=\left[\rho_{a} \rho_{b} H_{0}\right]^{\top}
$$

where

$$
\rho_{a}=\left[r_{a 1}, \theta_{a 1}, \ldots, r_{a M}, \theta_{a M}\right]^{\top}
$$

and

$$
\rho_{b}=\left[r_{b 1}, \theta_{b 1}, \ldots, r_{b N}, \theta_{b M}\right]^{\top} .
$$

The superscript $T$ denotes the transpose operation. An approximation error can be formulated as the difference between the actual amplitude response $M(x, \omega)$ and the desired amplitude or magnitude response $M_{d}(\omega)$ as

$$
e(x)=M(x, \omega)-M_{d}(\omega) .
$$

By sampling the error function $e\left(x, \omega_{i}\right)$, the actual and the desired amplitude responses $M\left(x, \omega_{i}\right)$ and $M_{d}(\omega)$ at frequencies $\omega 1, \omega_{2}, \ldots, \omega_{k}$, the column error vector is

$$
[E(x)]=\left[e_{1}(x), e_{2}(x), \ldots, e_{k}(x)\right]^{\top}
$$

where $e_{i}(x)=M\left(x, \omega_{i}\right)-M_{d}\left(\omega_{i}\right)$ and $\left\{\omega_{i}: i=1,2, \ldots, k\right\}$ is a dense set of frequencies which are distributed over in the pass-band and stop-band of the filter. A weighting or penalty error is included to control portions of the actual filter response curve that are most important to the filter response. This involves modifying the error to the form

$$
\bar{e}_{i}(x)=W(\omega)\left(M\left(x, \omega_{i}\right)-M_{d}\left(\omega_{i}\right)\right)
$$

where $W(\omega)$ is a weighting piece wise constant function over all frequency space, which is assigned a positive value greater than one or less than one to increase or decrease the magnitude approximation in a given band.

A recursive filter can be designed by finding a point $x=\hat{x}$ in (11) such that

$$
\bar{e}_{i}(x) \approx 0 \text { for } i=1,2, \ldots, k .
$$

Such a point can be obtained when solving the optimization problem by minimizing the error function $\bar{e}_{i}(x)$. The design of a recursive filter that approaches a specified response $M_{d}\left(\omega_{i}\right)$, can be performed by minimizing the error objective function in terms of $L_{p}$ norm error which is formulated as:

$$
\min _{x} \mu(x) \text { subject to } r_{a j} \leq 1-\delta
$$

where

$$
\mu(x)=\left\{\sum_{i=1}^{l} W_{i}(\omega)\left|e_{i}(x)\right|^{p}\right\}^{\frac{1}{p}}
$$

and

$$
e_{\max }(x)=\max _{1 \leq i \leq l}\left(e_{i}(x)\right) .
$$

The $p$ is a positive integer. The Taguchi technique is used to minimize $\mu(x)$ for increasing values of $p$. 


\subsection{Group-delay objective function}

The group delay is derived from the phase relation, as given in equation (4), and is defined as

$$
\tau(x, \omega)=\frac{\mathrm{d} \phi(x, \omega)}{\mathrm{d} \omega}
$$

where $\phi(x, \omega)$ is the phase response of the filter,

$$
\phi(x, \omega) \arg \left(H\left(e^{j \omega T}\right)\right)
$$

The group delay for a synthesized recursive filter is desired to be unchanged in the considered region one hand, in the other hand a minimum group delay is another required property needed in certain applications. in the present design, a constant group delay property is considered which is defined as

$$
\tau\left(x, \omega_{i}\right)=\tau_{c} \quad \text { for } \omega_{i} \in \omega_{p}
$$

where $\tau_{c}$ is constant, and $\omega_{p}$ is the passband region of the filter. In the present application, $\tau_{c}$ is an unknown but can be considered as the mean value over the passband region, which can be determined as

$$
\tau_{c, m}=\frac{1}{n_{k}} \sum_{i=1}^{n_{k}} \tau\left(\bar{x}_{m}, \omega_{i}\right) \text { for } \omega_{i} \in \omega_{p}
$$

where $\bar{x}_{m}$ is the optimal filter coefficient determined by minimizing the magnitude objective function for an m-th stability margin parameter.

The stability margin parameter is varied for discrete values, from which an optimal constant group delay is determined by minimizing the following objective error function

$$
\min _{m} E_{\tau}(m)=\frac{1}{\tau_{c, m}} \sum_{i=1}^{n_{k}}\left(\tau\left(\bar{x}_{m}, \omega_{i}\right)-\tau_{c, m}\right) \text { for } \omega_{i} \in \omega_{p}
$$

The multi-objective optimization problem is solved by discretizing the stability margin $\delta_{m}$ parameter, the magnitude optimization algorithm is used to generate the corresponding filter coefficients, in which basis the group delay is synthesized where a feasible and optimal solution can be obtained by minimizing the objective function (19). The final multiobjective design is obtained by minimizing the sum combination of the errors in equations (14) and (19).

\section{THE TAGUCHI OPTIMIZATION TECHNIQUE}

Compared with traditional optimization techniques, Taguchis optimization method is easy to implement and very efficient in reaching optimum solutions. Taguchis optimization method is developed based on the orthogonal array (OA) concept, which offers a systematic and efficient way to select design parameters. In addition, it reduces the number of tests required in the optimization process compared to GA or PSO $[54,55]$.

Taguchis method was developed based on the concept of the orthogonal array (OA), which can effectively reduce the number of tests required in a design process $[54,55]$. It provides an efficient way to choose the design parameters in an optimization procedure.

Before presenting the Taguchi procedure, it is worth understanding what OAs are and how are they generated $[54,55]$. Let $S$ be a set of s symbols or levels (the simplest symbols are integers $1,2,3 \ldots)$. A matrix $A$ of $N$ rows and $k$ columns with entries from $S$ is said to be an OA with $s$ levels and strength $t(0<t<k)$ if in every $N \times t$ subarray of $A$, each $t$-tuple based on $S$ appears exactly the same times as a row. The notation $\mathrm{OA}(N, k, s, t)$ is used to represent an OA.

\subsection{Initialization procedure}

The optimization procedure starts with the problem initialization, which includes the selection of a proper $O A$ and the design of a suitable fitness function. The selection of an $\operatorname{OA}(N, k, s, t)$ mainly depends on the number of optimization parameters. In general, to characterize the nonlinear effect, three levels $(s=3)$ are found sufficient for each input parameter. Usually, an OA with a strength of $2(t=2)$ is efficient for most problems because it results in a small number of rows in the array $[54,55]$.

\subsection{Design of input parameters}

The input parameters need to be selected to conduct the experiments. When the OA is used, the corresponding numerical values for the three levels of each input parameter should be determined.

In the first iteration, the value for level 2 is selected at the center of the optimization range. Values of levels 1 and 3 are calculated by subtracting/adding the value of level 2 with a variable called level difference (LD). The level difference in the first iteration (LD1) is determined by

$$
L D_{1}=\frac{\text { Max }- \text { Min }}{\text { Number of levels }+1}
$$

where Max is the upper bound of the optimization range and Min is the lower bound of the optimization range.

\subsection{Conduct Experiments and Build a Response Table}

After determining the input parameters, the fitness function for each experiment can be calculated. These results are then used to build a response table for the first iteration by averaging the fitness values for each parameter $\mathrm{n}$ and each level $m$ using the following equation

$$
F_{a v}=\frac{s}{N} \sum_{i, O A(i, n)=m} f_{i}
$$






Fig. 2. Magnitude filter response (SOOF)

As an example, consider that parameter $x$ in an $N$ dimensional problem has levels 1,2 and 3 as described earlier. With $s=2$ the fitness values are evaluated based on equation (7) for each level and hence a response table is constructed for each parameter that can be used to choose which level produces the best fitness value (minimum value).

\subsection{Identify Optimal Level Values and Conduct Confirmation Experiment}

Finding the largest fitness value ratio in each column can identify the optimal level for that parameter. When the optimal levels are identified, a confirmation experiment is performed using the combination of the optimal levels identified in the response table. This confirmation test is not repetitious because the OA-based experiment is a fractional factorial experiment, and the optimal combination may not be included in the experiment table. The fitness value obtained from the optimal combination is regarded as the fitness value of the current iteration.

\subsection{Reduce the Optimization Range}

If the results of the current iteration do not meet the termination criteria, the process is repeated in the next iteration. The optimal level values of the current iteration are used as central values (values of level 2) for the next iteration. To reduce the optimization range for a converged result, the $L D_{i}$ is multiplied with a reduced rate $(r r)$ to obtain $\mathrm{LD}_{i+1}$ for the $(i+1)^{\text {th }}$ iteration

$$
L D_{i+1}=r r \times L D_{i}=R R(i) \times L D_{i}
$$

where $R R(i)$ is called reduced function. When a constant $\mathrm{rr}$ is used, $R R(i)=r r^{i}$. The value of $r r$ can be set between 0.5 and 1 depending on the problem. The larger $r r$ is, the slower the convergence rate.

If $L D_{i}$ is a large value, and the central level value is located near the upper bound or lower bound of the optimization range, the corresponding value of level 1 or 3 may reside outside the optimization range. Therefore, a process of checking the level values is necessary to guarantee that all level values are located within the optimization range. A simple way is to use the boundary values directly.

\subsection{Check the Termination Criteria}

When the number of iterations is large, the level difference of each element becomes small from equation (22). Hence, the level values are close to each other and the fitness value of the next iteration is close to the fitness value of the current iteration. The following equation may be used as a termination criterion for the optimization procedure

$$
\frac{\mathrm{LD}_{i}}{\mathrm{LD}_{1}}<\text { converged value. }
$$

Usually, the converged value can be set between 0.001 and 0.01 depending on the problem. The iterative optimization process will be terminated if the design goal is achieved or if equation (23) is satisfied.

\section{RESULTS AND DISCUSSIONS}

The digital filter to be optimized is to be used to eliminate harmonics and sub-harmonics in a power network with a fundamental frequency of $50 \mathrm{~Hz}$. The filter is a bandpass type and is desired to satisfy the magnitude response to ideally pass only frequencies confined in the interval $\{45 \mathrm{~Hz}, 55 \mathrm{~Hz}\}$ while rejecting all other frequency content. The sampling frequency is taken to be $1800 \mathrm{~Hz}$. The filter is of order 10 and is hence composed of 5 cascaded SOSs. At start up, the filter is optimized to match the magnitude response specifications only. Next, more constraints are added to the optimization process including minimum and linear phase and constant group delay to enhance the designed filter performance.

\subsection{Single objective design}

The purpose of this part is to design a filter which only satisfies the magnitude response described earlier without considering any other performance criteria. The filter is designated single objective optimized filter (SOOF) hereafter. Figure 2 shows both the desired and the optimized magnitude response of the digital filter. It can be noted that the filter has good performance in the sense that it fulfils the requirements of magnitude response. Indeed, the filter response falls exactly within the desired response and it attenuates all other frequencies as the overall Sidelobe level is lower than $16 \mathrm{~dB}$.

However, the optimized filter is not of practical use as it suffers from drawbacks in the dynamic properties. First, as shown in Fig. 3, the time delay is in the order of $100 \mathrm{~ms}$ which is not suitable in the present applications as the requirements specify that the time delay should not exceed one cycle (20 ms). In addition, the group delay is not constant as shown in Fig. 4. Furthermore, the phase delay is nonlinear as shown in Fig. 5 and accordingly a non constant group delay. As a result, it is necessary to 


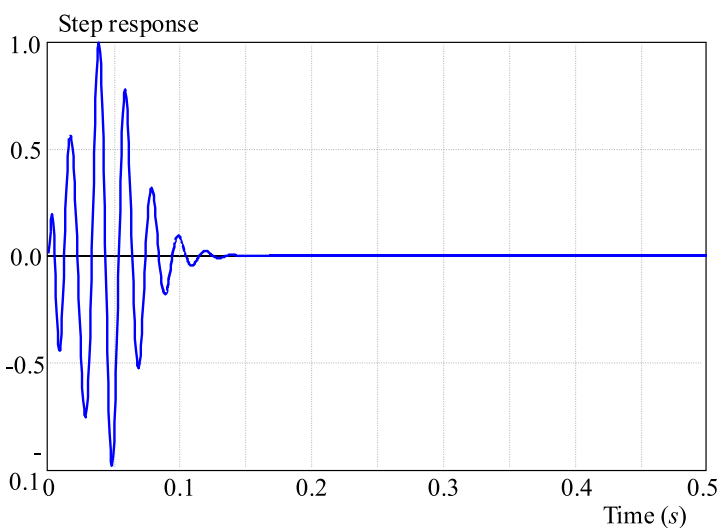

Fig. 3. Step response of the filter and time delay (about $100 \mathrm{~ms}$ ) (SOOF)



Fig. 5. Phase response of filter with non linear phase or non constant group delay (SOOF)

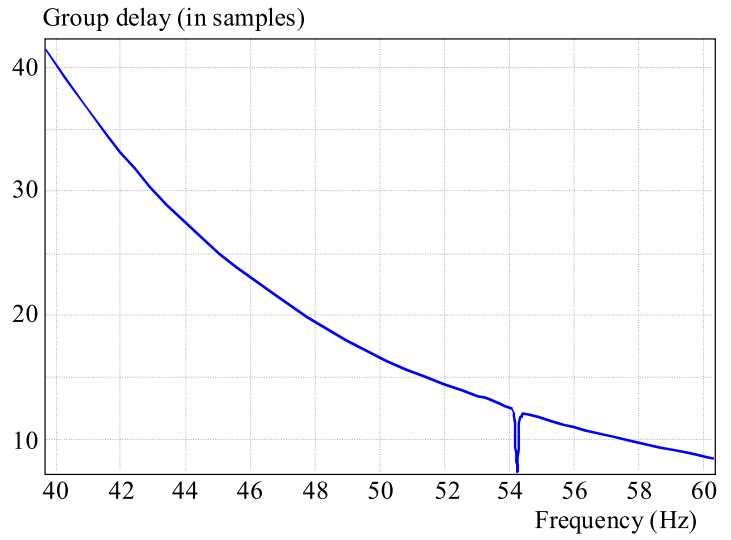

Fig. 4. Group delay response of single objective optimized filter (SOOF)



Fig. 6. Magnitude filter response (MOOF)

Table 1. Single-Objective Optimized Filter (SOOF) SOS coefficients and gains

\begin{tabular}{c|c|r|c|c|c|c|c|}
\hline Section & \multicolumn{3}{|c|}{ Numerator } & \multicolumn{3}{c|}{ Denominator } & Gain \\
\hline 1 & 1 & -1.9520345 & 0.9999998 & 1 & -1.8473812 & 0.8836000 & 0.0008977 \\
2 & 1 & -1.9999996 & 0.9999998 & 1 & -1.9999996 & 0.9999998 & 1 \\
3 & 1 & -1.9999996 & 0.9999998 & 1 & -1.8473812 & 0.8836000 & 1 \\
4 & 1 & -1.8851095 & 0.9999998 & 1 & -1.8473812 & 0.8836000 & 1 \\
5 & 1 & 1.9999998 & 0.9999998 & 1 & -1.8473812 & 0.8836000 & 1 \\
\hline
\end{tabular}

Table 2. Multi-Objective Optimized Filter (MOOF) SOS coefficients and gains

\begin{tabular}{c|c|r|r|r|r|c|c|}
\hline Section & \multicolumn{3}{|c|}{ Numerator } & \multicolumn{3}{c|}{ Denominator } & 0Gain \\
\hline 1 & 1 & -1.8293383 & 0.9999998 & 1 & -1.0914383 & 0.3540250 & 0.0124999 \\
2 & 1 & -1.4247566 & 0.9999998 & 1 & -1.0914383 & 0.3540250 & 1 \\
3 & 1 & -1.1469437 & 0.9999998 & 1 & -1.1899988 & 0.3540250 & 1 \\
4 & 1 & 0.2279814 & 0.9999998 & 1 & -1.0914383 & 0.3540250 & 1 \\
5 & 1 & -0.4675396 & -0.5324602 & 1 & -1.1137364 & 0.3540250 & 1 \\
\hline
\end{tabular}

include all the preceding performance criteria into the design process and hence, the problem becomes a multiobjective optimization task. The results are summarized in Table 1 where are presented the filter SOS coefficients and SOS gains.

\subsection{Multiobjective filter design}

The inclusion of the constant and minimum group delay in the optimization task besides magnitude response criterion produced a filter which satisfies almost all requirements. The filter compromises between these criteria 


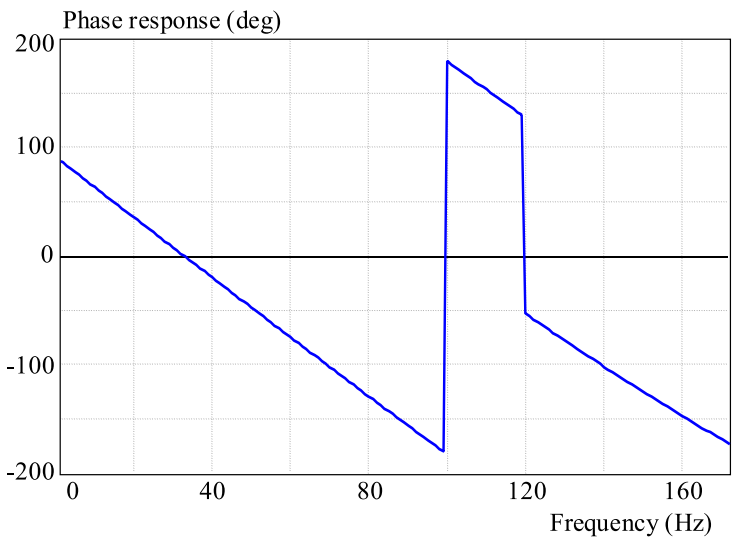

Fig. 7. Optimized filter phase response MOOF

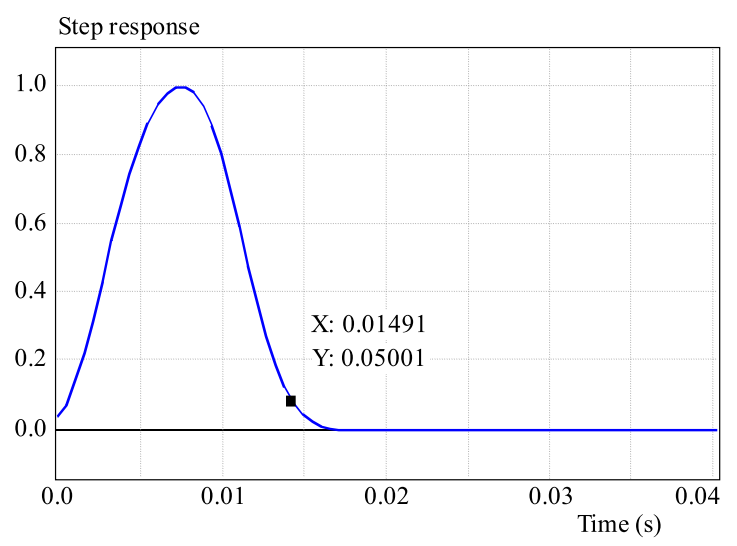

Fig. 9. Optimized filter step response and time delay (14.9 ms) $\mathrm{MOOF}$

to produce the best possible trade-off. The filter is thereafter labeled multiobjective optimized filter (MOOF). The magnitude response of the MOOF is shown in Fig. 6. The filter does not show good performance in terms of this characteristic compared to the SOOF. Indeed, the SOOF bandwidth is better than the MOOF which makes it having better selectivity.

Yet, the MOOF performs better in terms of the other criteria. In terms of phase and group responses, Figures 7 and 8 illustrate the phase and group delay responses. The filter is characterized by a linear phase with an almost



Fig. 10. Test signal: step of sinusoidal signal input and filter output response

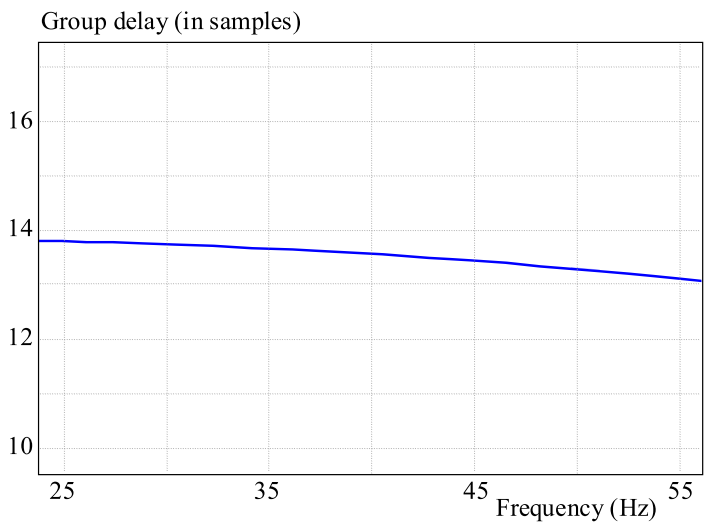

Fig. 8. Group delay response of optimized filter MOOF

constant group delay in the passband. Furthermore, the group delay is minimum producing a small time response as shown in Fig. 9. Indeed, the time delay of the MOOF is about $14.9 \mathrm{~ms}$ which conforms to the desired requirements. This is due to the fact that the stability margin or equivalently the pole radii have been taken into account in the optimization process and these latter have been lowered to a value of 0.595 instead of being closer to unity. Table 2 summarizes the SOS coefficients and gains of the MOOF.

To better assess the performance of the filter (MOOF), two tests have been performed. In the first, a step sinusoidal signal of $50 \mathrm{~Hz}$ is input to the filter and Fig. 10 shows both filter input and output waveforms. It is seen that the filter output matches exactly the input except for a phase shift and a time delay of less than one cycle. Hence, this filter proves to be practical for high speed measurement systems where the system accuracy is of great importance. In the second test, the previous step sinusoidal signal is corrupted with a DC offset, harmonic and subharmonic components. The subharmonic component is set to $25 \mathrm{~Hz}$ and the harmonics to 100, 150 and $200 \mathrm{~Hz}$. Both input and output signals are shown in Fig. 11. As it is clearly seen, the filter succeeded in eliminating the DC and harmonic components and mitigating the subharmonic component in a fast manner witnessed by the short time to start the filtering operation.



Fig. 11. Second test signal by considering a step sinusoidal signal mixed with harmonic and subharmonic components 


\section{CONCLUSION}

The application of the Taguchi optimization method to design a multiobjective filter digital has been considered in this paper. The objectives of the filter design were to match a desired magnitude response while having a minimum and linear phase. At start up, only magnitude response has been considered in the optimization task. The resulting filter was good in terms of this characteristic while it showed awful dynamic and phase performance. Next, the dynamic properties were included in the optimization algorithm to solve a multiobjective task. The Taguchi optimization method has succeeded in attaining the optimal design in terms of the previous requirements by achieving a compromise between them. The optimized filter has been tested and it showed good performance with required practical characteristics.

\section{REFERENCES}

[1] Antoniou, A.: Digital Filters: Analysis and Design, 2nd ed., McGraw Hill, New York, 1993.

[2] ANTOniou, A.: Digital Signal Processing: Signals, Systems and Filters, McGraw Hill, 2006.

[3] DUmitRescu, B.-NIEMisto, R.: Multistage IIR Filter Design using Convex Stability Domains Defined by Positive Realness, IEEE Trans. Signal Process 52 No. 4 (2004), 962-974.

[4] HO, C. Y.-F.-LING, B. W.-K.-CHI, Z.-W.-SHIKH-BAHAEI, M.-LIU, Y.-Q.-TEO, K.-L. : Design of Near-Allpass Strictly Stable Minimal-Phase Real Valued Rational IIR Filters, IEEE Trans. Circuits Syst. II, Exp. Briefs 55 No. 8 (2008), $781-785$.

[5] JIANG, A.-KWAN, H. K. : IIR Digital Filter Design with New Stability Constraint based on Argument Principle, IEEE Trans. Circuits Syst. I, Reg. Papers 56 No. 3 (2009), 583-593.

[6] JIANG, A.-KWAN, H. K.: Minimax Design of IIR Digital Filters using Iterative SOCP, IEEE Trans. Circuits Syst. I, Reg. Papers 57 No. 6 (2010), 1326-1337.

[7] JIANG, A.-KWAN, H. K.: Minimax Design of IIR Digital Filters using SDP Relaxation Techniques, IEEE Trans. Circuits Syst. I, Reg. Papers 57 No. 2 (2010), 378-390.

[8] LAI, X. P.: Optimal Design of Nonlinear-Phase FIR Filters with Prescribed Phase Error, IEEE Trans. Signal Process 57 No. 9 (2009), 3399-3410.

[9] LAI, X. P.-LIN, Z. P. : Minimax Design of IIR Digital Filters using a Sequential Constrained Least-Squares Method, IEEE Trans. Signal Process. 58 (2010), 3901-3906.

[10] LANG, M. C.: Least-Squares Design of IIR Filters with Prescribed Magnitude and Phase Responses and a Pole Radius Constraint, IEEE Trans. Signal Process 48 No. 11 (2000), 3109-3121.

[11] LU, W. S.: An Argument-Principle based Stability Criterion and Application to the Design of IIR Digital Filtersinbook Proc. IEEE Int. Symp. Circuits Syst., Island of Kos, Greece.

[12] LU, W. S.: Design of Stable Minimax IIR Digital Filters using Semidefinite Programming, Proc. IEEE Int. Symp. Circuits Syst., vol. 1, Geneva, Switzerland, 2000, pp. 355-358.

[13] LU, W. S. : Design of Recursive Digital Filters with Prescribed Stability Margin: A Parameterization Approach, IEEE Trans. Circuits Syst. II, Analog Digit. Signal Process 45 No. 9 (1998), 1289-1298.

[14] LU, W. S.-HINAmoto, T.: Optimal Design of IIR Digital Filters with Robust Stability using Conic Quadratic Programming Updates, IEEE Trans. Signal Process. 51 No. 6 (2003), 1581-1592.
[15] LANG, M. C.: Constrained Design of Digital Filters with Arbitrary Magnitude and Phase Responses, PhD Dissertation, Vienna Univ. Technol., Vienna, Austria, 1999.

[16] LU, W. S.-PEI, S. C.-TSENG, C. C.: A Weighted LeastSquares Method for the Design of Stable 1-D and 2-D IIR Digital Filters, IEEE Trans. Signal Process. 46 No. 1 (1998), 1-10.

[17] OMoifo, O. I.-Hinamoto, T. : Optimal Design of Stable Recursive Digital Filters using Unconstrained Optimization Methods, Proc. Int. Midwest Symp. Circuits Syst., Hiroshima, Japan 2, II-49II-52, 2004.

[18] PAN, S. T.: Design of Robust D-Stable IIR Filters using Genetic Algorithms with Embedded Stability Criterion, IEEE Trans. Signal Process. 57 No. 8 (2009), 3008-3016.

[19] QUelhas, M. F.-PETRAGLIA, A.: Digital Filter Design Optimization using Partial Cost Functions, Proc. IEEE Int. Symp. Circuits Syst., Taipei, Taiwan, 2009, pp. 285-288.

[20] SAAB, S.-LU, W. S.-ANTONIOU, A.: Design and Implementation of Lowpower IIR Digital Filter Systems, Proc. IEEE Int. Symp. Circuits Syst., Orlando, FL3, 1999, pp. 391-394.

[21] SANATHAnAN, C. K.-KOERnER, J.: Transfer Function Synthesis as a Ratio of Two Complex Polynomials, IEEE Trans. Autom. Control AC-8 No. 1 (1963), 56-58.

[22] TSENG, C. C.: Design of Stable IIR Digital Filter based on Least P-Power Error Criterion, IEEE Trans. Circuits Syst. I, Reg. Papers 51 No. 9 (2004), 1879-1888.

[23] TSEnG, C. C.-LEE, S. L.: Minimax Design of Stable IIR Digital Filter with Prescribed Magnitude and Phase Responses, IEEE Trans. Circuits Syst. I, Fundam. Theory Appl. 49 No. 4 (2002), 547-551.

[24] INUKAI, T. : A Unified Approach to Optimal Recursive Digital Filter Design, IEEE Trans. on Circuits and Systems CAS-27 No. 7 (1980), 646-649.

[25] CORTElazzo, G. LightneR, M. R. : Simultaneous Design in Both Magnitude and Group-Delay of IIR and FIR Filters based on Multiple Criterion Optimization, IEEE Trans. on Circuits and Systems 32 No. 5 (1984), 949-967.

[26] SULLIVAN, J. L.-ADAMS, J. W. : PCLS IIR Filters with Simultaneous Frequency Response Magnitude and Phase Related Specifications, Conference Record of the Thirty-First Asilomar; Conference on Signals, Systems and Computers1, Pacific Grove, California, 1997, pp. 705-709.

[27] LANG, M. C. : Least-Squares Design IIR Filters with Prescribed Magnitude and Phase Response and a Pole Radius Constraint, IEEE Trans. Signal Processing 48 No. 11 (2000), 3109-3121.

[28] LERTNIPHONPHUN, W.-McCLELLAN, J. H.: Unified Design Algorithm for Complex FIR and IIR Filters, Proceedings of the IEEE International Conference on Accoustics, Speech and Signal Processing, vol. 6, Salt Lake City, Utah, 2001, pp. 3801-3804.

[29] ZHANG, X.-IWAKURA, H.: Design of IIR Digital Allpass Filters based on Eigenvalue Problemjour IEEE Trans. on Acoustics, Speech, and Signal Processing.

[30] LANG, M.-LAAKSO, T. I. : Simple and Robust Method for the Design of Allpass Filters using Least-Squares Phase Error Criterion, IEEE Tarns. on Circuits and Systems II 41 No. 1 (1994), 40-48.

[31] IKEHARA, M.-FUNAISHI, M.-KURODA, H.: Design of Complex All-Pass Networks using Remez Algorithm, IEEE Tarns. on Circuits and Systems II 39 No. 8 (1992), 549-556.

[32] JING, Z.: A New Method for Digital Allpass Filter Design, IEEE Trans. on.

[33] SREERAM, V.-AGATHOKLIS, P.: Design of Linear-Phase IIR Filters via Impulse- Response Gramians, IEEE Trans. on Signal Processing 40 No. 2 (1992), 389-394.

[34] PENG, S. C.-CHEN, B. S.-CHIOU, B. W. : Simultaneous Design in Both Magnitude and Group-Delay of IIR and FIR Filters based on Multiple Criterion Optimization, IEE Proceedings G 139 No. 5 (1992), 586-590. 
[35] BELICZYNSKI, B.-KALE, I.-CAIN, G. D. : Approximation of FIR and IIR Digital Filters: an Algorithm based on Balanced Model Reduction, IEEE Trans. on Signal Processing 403 (1992), $532-542$.

[36] CORETlAzZO. C.-LIGHTNER, M. R. : Simultaneous Design in Both Magnitude and Group-Delay of IIR and FIR Filters Based on Multiple Criterion Optimization, IEEE Transactions on Acoustics, Speech and Signal Processing 5 (1984).

[37] LUTOVAA.: Design of Computationally Efficient Elliptic IIR Filters with a Reduced Number of Shift-and-Add Operations in Multipliers, IEEE Transactions on Signal processing 45 No. 10 (1997).

[38] SULLIVAN, J. L.-ADAMS, J. W. : PCLS IIR Digital Filters with Simultaneous Frequency Response Magnitude and Group Delay Specifications, IEEE Transactions on Signal Processing 46 No. 11 (1998).

[39] LANG: Least-Squares Method for Design of IIR Filters with Prescribed Magnitude and Phase Responses and a Pole Radius Constraint, IEEE Transactions on Signal Processing 48 No. 11 (2000).

[40] JOVANOVIC-DOLECEK, G.: Design of IIR Notch Filters with Maximally Flat or Equiripple Magnitude Characteristics, 14th Europeon Signal Processing Conference (EUSIPCO 2006), Florence, Italy, 2006.

[41] XI ZHANG: Design of Maximally Flat IIR Filters with Flat Group Delay Responses, Signal Processing 88 (2008), 1792-1800.

[42] RECIOUI, A.: Sidelobe Level Reduction in Linear Array Pattern Synthesis using Particle Swarm Optimization, J. of Optimization Theory and Applications 153 No. 2 (2012), 497-512, DOI 10.1007/s10957-011-9953-9.

[43] WANG, X.-MENG, X.-HE, Y.: A Novel Neural Networks-Based Approach for Designing FIR Filters, The Sixth World Congress on Intelligent Control and Automation, vol. 1, 2006, pp. 4029-4032.

[44] WANG, W. P.-ZHOU, L. F.-QIAN, J. X. : Fir Filter Design: Frequency sampling Filters By Particle Swarm Optimization Algorithm, Proc. international conference on machine learning and cybernetics, vol. 4, 2004, pp. 2332-2327.

[45] KRUSIENSKI, D. J.-JENKINS, W. K. : Particle Swarm Optimization for Adaptive IIR Filter Structures, Congress on Evolutionary Computation 1 (2004), 965-970.

[46] CHEN, H. C.-CHEN, O. T. : Particle Swarm Optimization incorporating a Preferential Velocity-Updating Mechanism and Its Application in IIR Filter Design, IEEE International Conference on Systems, Man and Cybernetics (2006), 1190-1195.

[47] STORN, R.: Differential Evolution Design of an IIR Filter, Proc. IEEE International Conference on Evolutionar Computation, 2006, pp. 268-273.

[48] STORN, R.: Designing Nonstandard Filters with Differential Evolution, Signal Processing Magazine. IEEE 22 (2005), 103-106.

[49] KARABOGA, N.: Digital Filter Design using Differential Evolution Algorithm, EURASIP Journal of Applied Signal Procesing 8 (2005)), 1269-1276.

[50] DERVIS KARABOGA: Simple and Global Optimization Algorithm for Engineering Problems, DE, Turk. J. Elec. Eng. 12 No. 1 (2004)

[51] TANG-KIM-FUNG MAN : Design and Optimization of IIR Filter Structure using Hierarchical Genetic Algorithms, IEEE Transactions on Industrial Electronics 45 No. 3 (1998).

[52] NURHAN KARABOGA: Design of Minimum Phase Digital IIR Filters by using Genetic Algorithm, Proceedings of the 6th Nordic Signal Processing Symposium - NORSIG, 2004
53] ABABNEH, J. I.-BATAINEH, M. H. : Linear Phase FIR Filter Design using Particle Swarm Optimization and Genetic Algorithms, Digital Signal Processing (2007), doi: 10.1016/j.dsp.2007.05.011..

54] WEI-CHUNG WENG-FAN YANG-ATEF ELSHERBENI : Electromagnetics and Antenna Optimization using Taguchis Method, Morgan and Claypool Publishers, 2007.

[55] WEI-CHUNG WENG et al : Linear Antenna Array Synthesis Using Taguchis Method: A Novel Optimization Technique in Electromagnetics, IEEE Transactions on Antennas and Propagation 55 No. 3 (Mar 2007).

Received 12 August 2012

Abderrahmane Ouadi was born in Rouiba, Algiers, Algeria. He received his engineering degree in 1989 at Bab Ezzouar University USTHB and Magister Degree with honors in Electrical Engineering from Institut National d'Electricite et d'Electronique" (INELEC), Boumerdes, Algeria, in 1995.Abderrahmane was a lecturer at INELEC from 1995 to 1999, he is continuing as a faculty member till date at the Institute of Electrical and Electronic Engineering, University of Boumerdes, Algeria. He is with a team research group of Signals and systems Lab since 2001. His main research focus is measurement systems and application of computer technology to power system monitoring, quality, protection and modeling of disturbances and transients in power systems. He has been a visiting researcher chercheur visiteur at BEAMS "Bio Electro-mechanical systems" energy group Laboratory, University Libre de Bruxelles, Belgium, during 2008-2010.

Hamid Bentarzi was born in 1965 in Legata, Boumerdes, Algeria. He received His Ingniorat and Magister degrees from Institut National d'Electricit et Electronique (INELEC), Boumerdes, in 1989 and 1992 respectively. Both are in applied Electronics. However, he Achieved his $\mathrm{PhD}$ degree at the Ecole Nationale Polytechnique, Algiers, in Micro-Electronic, in 2004. From 1993 to 1998 he was lecturer at INELEC, Boumerdes. Since 1998 he has been a member of the Engineering Science faculty at the University of Boumerdes, with activities centered upon control and protection systems. His current research interests include power systems protection and wide area measurement systems improvement using the recent developed technologies of communication. He has been author and co-author in more than 45 technical papers.

Abdelmadjid Recioui is an Associate Professor at the Institute of Electrical Engineering and Electronics University of Boumerdes, Algeria. He obtained a $\mathrm{PhD}$ degree in electrical and electronic engineering option telecommunications from the Institute of Electrical Engineering and Electronics, University of Boumerdes in 2011. He holds also Master (Magister) Electronic System Engineering degree which has been achieved at the Institute of Electrical Engineering and Electronics, University of Boumerdes in 2006. In June 2002, he finished his engineering studies at the institute of Electrical Engineering and Electronics, University of Boumerdes. He is a research Assistant at the laboratory signals and systems from January 2008 to Present in Laboratory: signals and systems, Inst. of Electrical Engineering and electronics, University of Boumerdes. His research interests include: Antennas, Wireless Communication Systems, antenna array synthesis and design, capacity enhancement, system optimization, smart antennas, power system protection, power system optimization, power system communications. 